\title{
SOME NEW RESULTS ON THE MOMENT GENERATING FUNCTION ORDER AND RELATED LIFE DISTRIBUTIONS
}

\author{
SHUHONG ZHANG, ${ }^{*}$ Lanzhou University \\ XIAOHU LI, ${ }^{* *}$ Xiamen University
}

\begin{abstract}
In this paper we study the moment generating function order and the new better than used in the moment generating function order $\left(\mathrm{NBU}_{\mathrm{MG}}\right)$ life distributions. A closure property of this order under an independent random sum is deduced, and stochastic comparisons among the block replacement policy, the age replacement policy, the complete repair policy, and the minimal repair policy of an $\mathrm{NBU}_{\mathrm{MG}}$ component are investigated.
\end{abstract}

Keywords: Age replacement; block replacement; complete repair; minimal repair; random sum

2010 Mathematics Subject Classification: Primary 62E10

Secondary $60 \mathrm{~K} 10$

\section{Introduction and preliminaries}

Stochastic orders and ageing properties play an important role in reliability theory, survival analysis, and other fields. Lots of stochastic orders and ageing properties have been studied in the literature; see, e.g. Shaked and Shanthikumar (2007), Marshall and Olkin (2007), Lai and Xie (2006), Müller and Stoyan (2002), and Barlow and Proschan (1981) for comprehensive discussions.

As is well known, Laplace transforms as well as Laplace transform orders are widely applied in engineering, economics, actuarial science, etc. In reliability theory, they are utilized to define ageing concepts; for example, Wang (1996), Belzunce et al (1999), and Yue and Cao (2001) independently studied $\mathrm{NBU}_{\mathrm{LT}}$ (new better than used in the Laplace transform order), which is characterized as a Laplace transform order between the residual lifetime and the total lifetime, and forms a nice extension to the NBU (new better than used) order. Klefsjö (1983) proposed the $\mathcal{L}$ class, which is characterized as the Laplace transform order between the lifetime and the exponential life with equal expectation, and yields an extension to the class HNBUE (harmonic new better than used in expectation). However, through an example of a life with failure rate tending to 0 as time goes to $\infty$, Klar (2002) showed that the $\mathcal{L}$ class may not be a reasonable notion for positive ageing. Subsequently, Klar and Müller (2003) introduced the moment generating function (MGF) order and studied the $\mathcal{M}$ class, which was proved there to be a more reasonable class of life distributions with positive ageing. Recently, Li (2004) further proved that the $\mathrm{NBU}_{\mathrm{MG}}$ class was closed under the increasing star-shaped transformations, and that the $\mathcal{M}$ class was closed under both convex linear combination and geometric compounding.

Received 26 February 2010; revision received 24 September 2010.

Supported by the National Natural Science Foundation of China (grant no. 10771090).

* Postal address: School of Mathematics and Statistics, Lanzhou University, Lanzhou 730000, P. R. China.

** Postal address: School of Mathematical Science, Xiamen University, Xiamen 361005, P. R. China.

Email address: xhli@1zu.edu.cn 
In this paper we will make a further study along this line of research. In Section 2 we investigate the closure property of the MGF order under a random sum of independent random lifetimes, which generalizes that of the independent and identical lifetimes in Klar and Müller (2003). In Section 3 we conduct stochastic comparisons among the block replacement policy, the age replacement policy, the complete repair policy, and the minimal repair policy of a component with $\mathrm{NBU}_{\mathrm{MG}}$ lifetime, and find that both the block replacement policy and the age replacement policy diminish the number of failures of the unit in the MGF order sense.

For ease of reference, let us recall some important concepts of stochastic orders and ageing properties, which will be discussed in the sequel. Throughout this paper, all random variables are implicitly assumed to be nonnegative with common left endpoint 0 and expectations are always assumed to be finite when used.

For two random variables $X$ and $Y$ with respective distributions $F$ and $G$ and survival functions $\bar{F}=1-F$ and $\bar{G}=1-G$, their respective MGFs are defined as, for all $s \geq 0$,

$$
\Psi_{X}(s)=\int_{0}^{\infty} \mathrm{e}^{s x} \mathrm{~d} F(x), \quad \Psi_{Y}(s)=\int_{0}^{\infty} \mathrm{e}^{s y} \mathrm{~d} G(y) .
$$

Based on the MGF, a stochastic order may be defined as follows.

Definition 1.1. (Klar and Müller (2003).) A random variable $Y$ is said to be larger than $X$ in the MGF order (denoted by $X \leq_{\mathrm{MGF}} Y$ ) if $\mathrm{E}\left[\mathrm{e}^{s Y}\right]$ is finite for some $s>0$ and $\Psi_{X}(s) \leq \Psi_{Y}(s)$ for all $s \geq 0$.

Let $X_{t}=[X-t \mid X>t]$ be the residual life of a unit with life $X$ at age $t \geq 0$. Then, it has the survival function $\bar{F}_{t}(x)=\bar{F}(t+x) / \bar{F}(t)$ for $x, t \geq 0$. By a comparison between the residual life and the total lifetime, quite a lot of ageing notions may be introduced based on various stochastic orders. In this paper we pay attention to the ageing notion based on the MGF order.

Definition 1.2. (Klar and Müller (2003), $L i$ (2004).) A random variable $X$ belongs to the class $\mathcal{M}$ if there exists some exponential random variable $Y$ such that $\mathrm{E} X=\mathrm{E} Y$ and $X \leq_{\mathrm{MGF}} Y ; X$ is said to be in the class $\mathrm{NBU}_{\mathrm{MG}}$ if $X_{t} \leq_{\mathrm{MGF}} X$ for all $t \geq 0$.

Both $\overline{\mathcal{M}}$ and NWU $\mathrm{NG}_{\mathrm{MG}}$ (new worse than used in the MGF order) may be defined by reversing the corresponding inequalities above. It is easy to verify that $X$ is $\mathrm{NBU}_{\mathrm{MG}}$ if and only if

$$
\int_{0}^{\infty} \mathrm{e}^{s x} \bar{F}(x+t) \mathrm{d} x \leq \bar{F}(t) \int_{0}^{\infty} \mathrm{e}^{s x} \bar{F}(x) \mathrm{d} x \quad \text { for all } t, s \geq 0,
$$

or, equivalently,

$$
\left(1-\mathrm{e}^{s t} \bar{F}(t)\right) \int_{0}^{\infty} \mathrm{e}^{s u} \bar{F}(u) \mathrm{d} u \leq \int_{0}^{t} \mathrm{e}^{s u} \bar{F}(u) \mathrm{d} u \quad \text { for all } t, s \geq 0 .
$$

\section{Closure under a random sum}

Let $M$ be the random number of shocks that occur up to time $t$, and let $X_{i}$ be the random damage inflicted by the $i$ th shock. Then $\sum_{i=1}^{M} X_{i}$ is the total damage that has accumulated by time $t$. Klar and Müller (2003) noted that the MGF order was closed under both the sum of independent components and the random sum of independent and identically distributed components. Theorem 2.1 below presents the closure property of this order under a random 
sum of independent but nonidentically distributed components; it asserts that, in the sense of MGF order, increasing either the number of shocks or the damage inflicted by each shock will increase the total damage accumulated.

Theorem 2.1. Suppose that $\left\{X_{i}\right\}_{i=1}^{\infty}$ and $\left\{Y_{i}\right\}_{i=1}^{\infty}$ are mutually independent random variables, and that $M$ and $N$ are integer-valued random variables independent of $\left\{X_{i}\right\}_{i=1}^{\infty}$ and $\left\{Y_{i}\right\}_{i=1}^{\infty}$. If $M \leq_{\mathrm{MGF}} N$ and there exists a sequence of independent and identical random variables $\left\{Z_{i}\right\}_{i=1}^{\infty}$ such that $X_{i} \leq_{\mathrm{MGF}} Z_{i} \leq_{\mathrm{MGF}} Y_{i}$ for $i \geq 1$, then $\sum_{i=1}^{M} X_{i} \leq_{\mathrm{MGF}} \sum_{i=1}^{N} Y_{i}$.

Proof. Define $\Phi_{M}(z)=\mathrm{E}\left[z^{M}\right]$ and $\Phi_{N}(z)=\mathrm{E}\left[z^{N}\right]$ with $z \geq 1$. Since $X_{i} \leq_{\mathrm{MGF}} Z_{i}$ for all $i \geq 1$, it holds that $\Psi_{X_{i}}(s) \leq \Psi_{Z_{i}}(s)$ for all $s \geq 0$, and, thus,

$$
\begin{aligned}
\mathrm{E}\left[\exp \left(s \sum_{i=1}^{M} X_{i}\right)\right] & =\sum_{n=0}^{\infty} \mathrm{P}(M=n) \mathrm{E}\left[\exp \left(s \sum_{i=1}^{M} X_{i}\right) \mid M=n\right] \\
& =\sum_{n=0}^{\infty} \mathrm{P}(M=n) \mathrm{E}\left[\exp \left(s \sum_{i=1}^{n} X_{i}\right)\right] \\
& =\sum_{n=0}^{\infty} \mathrm{P}(M=n) \prod_{i=1}^{n} \Psi_{X_{i}}(s) \\
& \leq \sum_{n=0}^{\infty} \mathrm{P}(M=n) \prod_{i=1}^{n} \Psi_{Z_{i}}(s) \\
& =\sum_{n=0}^{\infty} \mathrm{P}(M=n) \Psi_{Z_{1}}^{n}(s) \\
& =\Phi_{M}\left(\Psi_{Z_{1}}(s)\right) .
\end{aligned}
$$

Similarly, $Z_{i} \leq_{\mathrm{MGF}} Y_{i}$ for all $i \geq 1$ implies that, for all $s \geq 0$,

$$
\Phi_{N}\left(\Psi_{Z_{1}}(s)\right) \leq \mathrm{E}\left[\exp \left(s \sum_{i=1}^{N} Y_{i}\right)\right] .
$$

On the other hand, it is evident that $M \leq_{\text {MGF }} N$ if and only if $\Phi_{N}(z) \geq \Phi_{M}(z)$ for all $z \geq 1$. Combining (2.1) and (2.2), we have, for all $s \geq 0$,

$$
\mathrm{E}\left[\exp \left(s \sum_{i=1}^{M} X_{i}\right)\right] \leq \mathrm{E}\left[\exp \left(s \sum_{i=1}^{N} Y_{i}\right)\right]
$$

This is just $\sum_{i=1}^{M} X_{i} \leq_{\mathrm{MGF}} \sum_{i=1}^{N} Y_{i}$, completing the proof.

Example 2.1 below shows that the condition $X_{i} \leq_{\mathrm{MGF}} Z_{i} \leq_{\mathrm{MGF}} Y_{i}$ in Theorem 2.1 cannot be relaxed to $X_{i} \leq_{\mathrm{MGF}} Y_{i}$.

Example 2.1. Suppose that $M$ and $N$ have distributions

$$
\begin{array}{lll}
\mathrm{P}(M=1)=0.1, & \mathrm{P}(M=2)=0.3, & \mathrm{P}(M=3)=0.6, \\
\mathrm{P}(N=1)=0.2, & \mathrm{P}(N=2)=0.1, & \mathrm{P}(N=3)=0.7 .
\end{array}
$$


It is easy to evaluate

$$
\Phi_{M}(z)=0.1 z+0.3 z^{2}+0.6 z^{3} \quad \text { and } \Phi_{N}(z)=0.2 z+0.1 z^{2}+0.7 z^{3} \quad \text { for } z \geq 1,
$$

and, therefore, $\Phi_{N}(z)-\Phi_{M}(z)=0.1 z\left(z^{2}-2 z+1\right) \geq 0$ for any $z \geq 1$. Hence, $\Phi_{M}(z) \leq \Phi_{N}(z)$ for any $z \geq 1$. That is, $M \leq_{\mathrm{MGF}} N$.

Consider two independent binary sequences $\left\{X_{i}\right\}_{i=1}^{\infty}$ and $\left\{Y_{i}\right\}_{i=1}^{\infty}$ with

$$
\mathrm{P}\left(X_{i}=1\right)=p_{i}=1-\mathrm{P}\left(X_{i}=0\right) \quad \text { and } \quad \mathrm{P}\left(Y_{i}=1\right)=q_{i}=1-\mathrm{P}\left(Y_{i}=0\right)
$$

such that $0 \leq p_{i} \leq q_{i} \leq 1$ for $i=1,2, \ldots$. It is easy to verify that

$$
\Phi_{X_{i}}(z)=(z-1) p_{i}+1 \leq(z-1) q_{i}+1=\Phi_{Y_{i}}(z) \text { for } z \geq 1,
$$

which reduces to $X_{i} \leq_{\mathrm{MGF}} Y_{i}$ for $i=1,2, \ldots$ It is obvious that, for $p_{1}=q_{1}=0.9$, $p_{2}=q_{2}=0.8, p_{3}=0.1$, and $q_{3}=0.11$, there are no independent and identical $Z_{1}, Z_{2}$, and $Z_{3}$ such that $X_{i} \leq_{\mathrm{MGF}} Z_{i} \leq_{\mathrm{MGF}} Y_{i}$ for $i=1,2,3$.

On the other hand, for $z \geq 1$,

$$
\begin{aligned}
\mathrm{E}\left[z^{\sum_{i=1}^{M} X_{i}}\right] & =\sum_{n=1}^{3} \mathrm{P}(M=n) \mathrm{E}\left[z^{\sum_{i=1}^{M} X_{i}} \mid M=n\right] \\
& =\sum_{n=1}^{3} \mathrm{P}(M=n) \mathrm{E}\left[z^{\sum_{i=1}^{n} X_{i}}\right] \\
& =\sum_{n=1}^{3} \mathrm{P}(M=n) \prod_{i=1}^{n} \Phi_{X_{i}}(z) \\
& =0.1 \Phi_{X_{1}}(z)+0.3 \Phi_{X_{1}}(z) \Phi_{X_{2}}(z)+0.6 \Phi_{X_{1}}(z) \Phi_{X_{2}}(z) \Phi_{X_{3}}(z) .
\end{aligned}
$$

Similarly, for $z \geq 1$,

$$
\mathrm{E}\left[z^{\sum_{i=1}^{N} Y_{i}}\right]=0.2 \Phi_{Y_{1}}(z)+0.1 \Phi_{Y_{1}}(z) \Phi_{Y_{2}}(z)+0.7 \Phi_{Y_{1}}(z) \Phi_{Y_{2}}(z) \Phi_{Y_{3}}(z) .
$$

Thus, we have

$$
\left.\mathrm{E}\left[z^{\sum_{i=1}^{M} X_{i}}\right]\right|_{z=2}=3.4732>3.37934=\left.\mathrm{E}\left[z^{\sum_{i=1}^{N} Y_{i}}\right]\right|_{z=2} .
$$

This invalidates $\sum_{i=1}^{M} X_{i} \leq_{\text {MGF }} \sum_{i=1}^{N} Y_{i}$.

To end this section, let us present an interesting application of Theorem 2.1.

Consider a device subject to a sequence of shocks arriving at random according to a counting process with either interarrivals $\left\{X_{i}\right\}_{i=1}^{\infty}$ or interarrivals $\left\{Y_{i}\right\}_{i=1}^{\infty}$ with $\mathrm{E} X_{i}=\mathrm{E} Y_{i}$ for $i=$ $1,2, \ldots$ Let $N_{1}$ and $N_{2}$ be the corresponding random numbers of shocks from which the device may survive. Note that $N_{1}$ and $N_{2}$ are assumed to be independent of the interarrival times of the shocks. Then the corresponding lifetimes of the device are $L_{1}=\sum_{i=1}^{N_{1}} X_{i}$ and $L_{2}=\sum_{i=1}^{N_{2}} Y_{i}$.

Corollary 2.1. Suppose that $X_{i} \in \mathcal{M}$ and $Y_{i} \in \overline{\mathcal{M}}$ for $i=1,2, \ldots$ If $N_{1} \leq_{\mathrm{MGF}} N_{2}$ then $L_{1} \leq_{\text {MGF }} L_{2}$.

Proof. Since $X_{i} \in \mathcal{M}, Y_{i} \in \overline{\mathcal{M}}$, and $\mathrm{E} X_{i}=\mathrm{E} Y_{i}=\mu$ for $i=1,2, \ldots$, it holds that $X_{i} \leq_{\mathrm{MGF}} Z_{i} \leq_{\mathrm{MGF}} Y_{i}$; here the independent and identical $Z_{i}$ s have exponential distribution with mean $\mu$. Thus, the desired result follows immediately from Theorem 2.1. 


\section{Repair and replacement policies}

In this section we present stochastic comparisons among the block replacement policy, the age replacement policy, the complete repair policy, and the minimal repair policy of a component with $\mathrm{NBU}_{\mathrm{MG}}$ life distribution. In what follows, the distribution of that component will be denoted by $F$.

In the complete repair policy, a failed unit is replaced with a new identical unit, and, thus, a complete repair policy is characterized as a renewal process $\{N(t), t \geq 0\}$ with the time of interarrivals being the random lifetime of the component. We denote by $N(t), \tau_{i}$, and $T_{i}$ the number of renewals in $[0, t]$, the time interval between the $(i-1)$ th and $i$ th renewals, and the arrival time of the $i$ th renewal, respectively. In the minimal repair policy, a failed unit is repaired to its condition just prior to failure. For a minimal repair policy, denote by $N^{(m)}(t), \tau_{i}^{(m)}$, and $T_{i}^{(m)}$ the number of minimal repairs up to time $t$, the time interval between the $(i-1)$ th and $i$ th minimal repair, and the arrival time of the $i$ th minimal repair, respectively. It is well known that $\left\{N^{(m)}(t), t \geq 0\right\}$ is a nonhomogeneous Poisson process.

Under a block replacement policy, a replacement is made whenever a failure occurs at specified times $k \ell$ with $\ell \geq 0$ and $k=1,2, \ldots$ Let $\left\{N^{(b)}(t, \ell), t \geq 0\right\}$ be the counting process related to the number of failures under a block replacement policy with planned replacement times $k \ell, k=1,2, \ldots$. Denote by $N^{(b)}(t, \ell), \tau_{i}^{(b)}$, and $T_{i}^{(b)}$ the number of failures in $[0, t]$, the time interval between the $(i-1)$ th and $i$ th failures, and the arrival time of the $i$ th failure under a block replacement policy, respectively. In the context of an age replacement policy, a unit is either replaced upon failure or upon reaching a specified age $\ell$, depending on which event occurs first. In a similar way, denote by $N^{(a)}(t, \ell), \tau_{i}^{(a)}$, and $T_{i}^{(a)}$ the number of failures in $[0, t]$, the time interval between the $(i-1)$ th and $i$ th failure, and the arrival time of the $i$ th failure under an age replacement policy, respectively. It should be noted here that the quantities $N^{(b)}(t, \ell)$ and $N^{(a)}(t, \ell)$ do not count planned replacements, only replacements due to failures.

In the literature, some stochastic orders have been employed to compare counting processes. For example, Yue and Cao (2001) compared counting processes in the Laplace transform order, and Belzunce et al. (2005) compared counting processes in terms of the increasing convex order. Here, we focus on comparing counting processes in terms of the MGF order.

Definition 3.1. Let $\left\{N_{1}(t), t \geq 0\right\}$ and $\left\{N_{2}(t), t \geq 0\right\}$ be two counting processes, with arrival times $\left\{R_{n}, n \geq 1\right\}$ and $\left\{S_{n}, n \geq 1\right\}$, respectively. If $R_{n} \geq_{\mathrm{MGF}} S_{n}$ for all $n \geq 1$, or, equivalently,

$$
\int_{0}^{\infty} \mathrm{e}^{s t} \mathrm{P}\left(R_{n}>t\right) \mathrm{d} t \geq \int_{0}^{\infty} \mathrm{e}^{s t} \mathrm{P}\left(S_{n}>t\right) \mathrm{d} t, \quad s>0, n=1,2, \ldots,
$$

then $\left\{N_{1}(t), t \geq 0\right\}$ is said to be smaller than $\left\{N_{2}(t), t \geq 0\right\}$ in the MGF order, denoted as $N_{1}(t) \leq_{\text {MGF }} N_{2}(t)$.

In light of the equivalence between $\left\{R_{n}>t\right\}$ and $\left\{N_{1}(t)<n\right\},(3.1)$ may be rephrased as

$$
\int_{0}^{\infty} \mathrm{e}^{s t} \mathrm{P}\left(N_{1}(t)<n\right) \mathrm{d} t \geq \int_{0}^{\infty} \mathrm{e}^{s t} \mathrm{P}\left(N_{2}(t)<n\right) \mathrm{d} t \quad \text { for all } s>0 \text { and } n=1,2, \ldots
$$

When comparing between the complete repair policy and the minimal repair policy, Block et al. (1990) proved that $N(t) \leq_{\mathrm{st}} N^{(m)}(t)$ for $t \geq 0$ if the underlying life distribution $F$ is NBU. Here, we present a parallel version for $\mathrm{NBU}_{\mathrm{MG}}$ life distributions.

Theorem 3.1. If the underlying distribution $F$ is $N B U_{M G}$ then $N(t) \leq_{\mathrm{MGF}} N^{(m)}(t)$ for all $t \geq 0$. 
Proof. By Definition 3.1, we only need to prove that $T_{n} \geq_{\mathrm{MGF}} T_{n}^{(m)}$, or, equivalently, $\sum_{i=1}^{n} \tau_{i} \geq_{\text {MGF }} \sum_{i=1}^{n} \tau_{i}^{(m)}$ for $n=1,2, \ldots$. In view of Theorem 2.2 of Klar and Müller (2003), it suffices to show that $\tau_{i} \geq_{\mathrm{MGF}} \tau_{i}^{(m)}$ for all $i=1,2, \ldots$.

Let us denote by $G_{j}(t), t \geq 0$, the distribution function of $\tau_{j}^{(m)}, j=1,2, \ldots$. Obviously, $G_{1}=F$. When $i>1$, the following recurrent relations are valid for $t \geq 0$ :

$$
\begin{aligned}
G_{i}(t) & =\mathrm{P}\left(\tau_{i}^{(m)}>t\right) \\
& =\int_{0}^{\infty} \cdots \int_{0}^{\infty} \mathrm{P}\left(\tau_{i}^{(m)}>t \mid \tau_{1}^{(m)}=t_{1}, \ldots, \tau_{i-1}^{(m)}=t_{i-1}\right) \mathrm{d} G_{1}\left(t_{1}\right) \cdots \mathrm{d} G_{i-1}\left(t_{i-1}\right) \\
& =\int_{0}^{\infty} \cdots \int_{0}^{\infty} \frac{\bar{F}\left(t+\sum_{j=1}^{i-1} t_{j}\right)}{\bar{F}\left(\sum_{j=1}^{i-1} t_{j}\right)} \mathrm{d} G_{1}\left(t_{1}\right) \cdots \mathrm{d} G_{i-1}\left(t_{i-1}\right) .
\end{aligned}
$$

Since $F$ is $\mathrm{NBU}_{\mathrm{MG}}$, by (1.1), we have, for all $s \geq 0$,

$$
\begin{aligned}
\int_{0}^{\infty} \mathrm{e}^{s t} \mathrm{P}\left(\tau_{i}^{(m)}>t \mid \tau_{1}^{(m)}=t_{1}, \ldots, \tau_{i-1}^{(m)}=t_{i-1}\right) \mathrm{d} t & =\int_{0}^{\infty} \mathrm{e}^{s t} \frac{\bar{F}\left(t+\sum_{j=1}^{i-1} t_{j}\right)}{\bar{F}\left(\sum_{j=1}^{i-1} t_{j}\right)} \mathrm{d} t \\
& \leq \int_{0}^{\infty} \mathrm{e}^{s t} \bar{F}(t) \mathrm{d} t
\end{aligned}
$$

Consequently,

$$
\begin{aligned}
\int_{0}^{\infty} \mathrm{e}^{s t} G_{i}(t) \mathrm{d} t & =\int_{0}^{\infty} \cdots \int_{0}^{\infty} \int_{0}^{\infty} \mathrm{e}^{s t} \mathrm{P}\left(\tau_{i}^{(m)}>t \mid \tau_{1}^{(m)}=t_{1}, \ldots, \tau_{i-1}^{(m)}=t_{i-1}\right) \\
& \times \mathrm{d} t \mathrm{~d} G_{1}\left(t_{1}\right) \cdots \mathrm{d} G_{i-1}\left(t_{i-1}\right) \\
& \leq \int_{0}^{\infty} \cdots \int_{0}^{\infty} \int_{0}^{\infty} \mathrm{e}^{s t} \bar{F}(t) \mathrm{d} t \mathrm{~d} G_{1}\left(t_{1}\right) \cdots \mathrm{d} G_{i-1}\left(t_{i-1}\right) \\
& =\int_{0}^{\infty} \mathrm{e}^{s t} \bar{F}(t) \mathrm{d} t
\end{aligned}
$$

That is, $\tau_{i}^{(m)} \leq_{\text {MGF }} \tau_{i}, i=1, \ldots, n$. This completes the proof.

In the literature, there are several related results for comparing between the block replacement policy and the corresponding renewal process. For example, Marshall and Proschan (1972) showed that $F$ is NBU if and only if $N(t) \geq_{\text {st }} N^{(b)}(t, \ell)$ for $t \geq 0$ and $\ell \geq 0$. Yue and Cao (2001) proved that $F$ is $\mathrm{NBU}_{\mathrm{LT}}$ if and only if $N(t) \geq_{\mathrm{LT}} N^{(b)}(t, \ell)$ for $t \geq 0$ and $\ell \geq 0$. Belzunce et al. (2005) proved that if $F$ is NBUC (new better than used in the increasing convex order) then $N(t) \leq_{\text {icx }} N^{(b)}(t, \ell)$ for $t \geq 0$ and $\ell \geq 0$. Recently, Belzunce et al. (2006) proved that if $F$ is NBUE (new better than used in expectation) then $\mathrm{E} T_{i} \leq \mathrm{E} T_{i}^{(b)}$ for $i=1,2, \ldots$. The forementioned ' $\leq_{\mathrm{st}}$ ', ' $\leq_{\mathrm{LT}}$ ', and ' $\leq_{\mathrm{icx}}$ ' denote the usual stochastic order, the Laplace transform order, and the increasing convex order, respectively. For more on stochastic orders, the reader is referred to Shaked and Shanthikumar (2007). A parallel version for $\mathrm{NBU}_{\mathrm{MG}}$ life distributions is presented in Theorem 3.2 below, which states that, when the underlying distribution is $\mathrm{NBU}_{\mathrm{MG}}$, the block replacement policy reduces the number of failures of the unit in any particular time interval $[0, t], 0<t<\infty$, in the sense of the MGF order.

Theorem 3.2. The underlying distribution $F$ is $N B U_{M G}$ if and only if $N(t) \geq_{\mathrm{MGF}} N^{(b)}(t, \ell)$ for all $t, \ell \geq 0$. 
Proof. Necessity. Similarly to the proof of Theorem 3.1, it suffices to show that $\tau_{i} \leq_{\mathrm{MGF}} \tau_{i}^{(b)}$ for all $i=1,2, \ldots$.

Denote by $H_{j}(t), t \geq 0$, the distribution function of $\tau_{j}^{(b)}, j=1,2, \ldots$. Obviously, when $i=1$,

$$
H_{1}(t)=\bar{F}^{k}(\ell) \bar{F}(t-k \ell) \quad \text { for } k \ell \leq t<(k+1) \ell \text { and } k=0,1,2, \ldots
$$

Thus, we have, for any $s \geq 0$,

$$
\int_{0}^{\infty} \mathrm{e}^{s t} H_{1}(t) \mathrm{d} t=\sum_{k=0}^{\infty} \int_{k \ell}^{(k+1) \ell} \mathrm{e}^{s t}[\bar{F}(\ell)]^{k} \bar{F}(t-k \ell) \mathrm{d} t=\sum_{k=0}^{\infty}\left[\mathrm{e}^{s \ell} \bar{F}(\ell)\right]^{k} \int_{0}^{\ell} \mathrm{e}^{s t} \bar{F}(t) \mathrm{d} t .
$$

If $\mathrm{e}^{s \ell} \bar{F}(\ell) \geq 1$, it is obviously true that

$$
\sum_{k=0}^{\infty}\left[\mathrm{e}^{s \ell} \bar{F}(\ell)\right]^{k} \int_{0}^{\ell} \mathrm{e}^{s t} \bar{F}(t) \mathrm{d} t \geq \int_{0}^{\infty} \mathrm{e}^{s t} \bar{F}(t) \mathrm{d} t
$$

If $\mathrm{e}^{s \ell} \bar{F}(\ell)<1$, from (1.2), it follows that

$$
\begin{aligned}
\sum_{k=0}^{\infty}\left[\mathrm{e}^{s \ell} \bar{F}(\ell)\right]^{k} \int_{0}^{\ell} \mathrm{e}^{s t} \bar{F}(t) \mathrm{d} t & =\frac{1}{1-\mathrm{e}^{s \ell} \bar{F}(\ell)} \int_{0}^{\ell} \mathrm{e}^{s t} \bar{F}(t) \mathrm{d} t \\
& \geq \frac{1}{1-\mathrm{e}^{s \ell} \bar{F}(\ell)}\left(1-\mathrm{e}^{s \ell} \bar{F}(\ell)\right) \int_{0}^{\infty} \mathrm{e}^{s t} \bar{F}(t) \mathrm{d} t \\
& =\int_{0}^{\infty} \mathrm{e}^{s t} \bar{F}(t) \mathrm{d} t
\end{aligned}
$$

Therefore, we have $\tau_{1} \leq_{\mathrm{MGF}} \tau_{1}^{(b)}$.

When $i>1$, it holds that, for any $t \geq 0$,

$$
H_{i}(t)=\int_{0}^{\infty} \cdots \int_{0}^{\infty} \mathrm{P}\left(\tau_{i}^{(b)}>t \mid \tau_{1}^{(b)}=t_{1}, \ldots, \tau_{i-1}^{(b)}=t_{i-1}\right) \mathrm{d} H_{1}\left(t_{1}\right) \cdots \mathrm{d} H_{i-1}\left(t_{i-1}\right) .
$$

Note that $\tau_{i} \stackrel{\text { st }}{=} \tau_{1}$ and $\left(\tau_{i}^{(b)} \mid \tau_{1}^{(b)}=t_{1}, \ldots, \tau_{i-1}^{(b)}=t_{i-1}\right) \stackrel{\text { st }}{=} \tau_{1}^{\left(b^{\prime}\right)}$ (here $\stackrel{\text { st }}{=}$ means that both sides have the same distribution), the first unplanned replacement time of the block replacement counting process with planned replacement times $\left\{k \ell-\sum_{j=1}^{i-1} t_{j}, k \ell>\sum_{j=1}^{i-1} t_{j}\right\}$ (see Block et al. (1990)). We have, for any $s \geq 0$,

$$
\begin{aligned}
\int_{0}^{\infty} \mathrm{e}^{s t} H_{i}(t) \mathrm{d} t & =\int_{0}^{\infty} \cdots \int_{0}^{\infty} \int_{0}^{\infty} \mathrm{e}^{s t} \mathrm{P}\left(\tau_{1}^{\left(b^{\prime}\right)}>t\right) \mathrm{d} t \mathrm{~d} H_{1}\left(t_{1}\right) \cdots \mathrm{d} H_{i-1}\left(t_{i-1}\right) \\
& \geq \int_{0}^{\infty} \cdots \int_{0}^{\infty} \int_{0}^{\infty} \mathrm{e}^{s t} \mathrm{P}\left(\tau_{1}>t\right) \mathrm{d} t \mathrm{~d} H_{1}\left(t_{1}\right) \cdots \mathrm{d} H_{i-1}\left(t_{i-1}\right) \\
& =\int_{0}^{\infty} \mathrm{e}^{s t} \mathrm{P}\left(\tau_{i}>t\right) \mathrm{d} t .
\end{aligned}
$$

That is, $\tau_{i} \leq_{\mathrm{MGF}} \tau_{i}^{(b)}$ for any $i>1$.

Sufficiency. Suppose that $N(t) \geq_{\mathrm{MGF}} N^{(b)}(t, \ell)$ for all $t \geq 0$. By (1.2), we only need to prove that

$$
\left(1-\mathrm{e}^{s \ell} \bar{F}(\ell)\right) \int_{0}^{\infty} \mathrm{e}^{s t} \bar{F}(t) \mathrm{d} t \leq \int_{0}^{\ell} \mathrm{e}^{s t} \bar{F}(t) \mathrm{d} t \quad \text { for all } s, \ell \geq 0 .
$$


The proof is straightforward for the case in which $\mathrm{e}^{s \ell} \bar{F}(\ell) \geq 1$, and so we focus on the case in which $\mathrm{e}^{s \ell} \bar{F}(\ell)<1$.

Since $N(t) \geq_{\text {MGF }} N^{(b)}(t, \ell)$ for any $t \geq 0$, we have, for any $s \geq 0$,

$$
\int_{0}^{\infty} \mathrm{e}^{s t} \mathrm{P}(N(t)<1) \mathrm{d} t \leq \int_{0}^{\infty} \mathrm{e}^{s t} \mathrm{P}\left(N^{(b)}(t, \ell)<1\right) \mathrm{d} t,
$$

which is equivalent to

$$
\int_{0}^{\infty} \mathrm{e}^{s t} \mathrm{P}(N(t)=0) \mathrm{d} t \leq \int_{0}^{\infty} \mathrm{e}^{s t} \mathrm{P}\left(N^{(b)}(t, \ell)=0\right) \mathrm{d} t .
$$

Since $\mathrm{P}(N(t)=0)=\bar{F}(t)$ for any $t \geq 0$ and

$$
\mathrm{P}\left(N^{(b)}(t, \ell)=0\right)=\bar{F}^{k}(\ell) \bar{F}(t-k \ell)
$$

for $k \ell \leq t<(k+1) \ell$ and $k=0,1,2, \ldots$, it follows that

$$
\int_{0}^{\infty} \mathrm{e}^{s t} \mathrm{P}(N(t)=0) \mathrm{d} t=\int_{0}^{\infty} \mathrm{e}^{s t} \bar{F}(t) \mathrm{d} t
$$

and

$$
\begin{aligned}
\int_{0}^{\infty} \mathrm{e}^{s t} \mathrm{P}\left(N^{(b)}(t, \ell)=0\right) \mathrm{d} t & =\sum_{k=0}^{\infty}\left[\mathrm{e}^{s \ell} \bar{F}(\ell)\right]^{k} \int_{0}^{\ell} \mathrm{e}^{s t} \bar{F}(t) \mathrm{d} t \\
& =\frac{1}{1-\mathrm{e}^{s \ell} \bar{F}(\ell)} \int_{0}^{\ell} \mathrm{e}^{s t} \bar{F}(t) \mathrm{d} t .
\end{aligned}
$$

As a result, we have

$$
\int_{0}^{\infty} \mathrm{e}^{s t} \bar{F}(t) \mathrm{d} t \leq \frac{1}{1-\mathrm{e}^{s \ell} \bar{F}(\ell)} \int_{0}^{\ell} \mathrm{e}^{s t} \bar{F}(t) \mathrm{d} t, \quad s, \ell \geq 0 .
$$

Therefore, (3.2) is validated and $F$ is $\mathrm{NBU}_{\mathrm{MG}}$.

Block et al. (1990) proved that $N^{(m)}(t) \geq_{\mathrm{st}} N^{(b)}(t, \ell)$ for all $t \geq 0$ and $\ell \geq 0$ if the underlying distribution is NBU. Block et al. (1993) further showed that the underlying distribution is NBU if and only if $N^{(m)}(t) \geq_{\text {st }} N^{(b)}(t, \ell)$ for all $t \geq 0$ and $\ell \geq 0$. In combination with Theorem 3.1 and Theorem 3.2, we immediately reach the following corollary. It is still an open problem whether this conclusion may be strengthened as an equivalent characterization of $\mathrm{NBU}_{\mathrm{MG}}$.

Corollary 3.1. If the underlying distribution $F$ is $N B U_{M G}$ then $N^{(m)}(t) \geq_{\mathrm{MGF}} N^{(b)}(t, \ell)$ for all $t, \ell \geq 0$.

A block replacement policy $N^{(b)}(t, \ell)$ is said to be a refinement of another replacement policy $N^{(b)}\left(t, \ell^{\prime}\right)$ if $\{k \ell\}_{k=1}^{\infty} \supset\left\{k \ell^{\prime}\right\}_{k=1}^{\infty}$. When one block replacement policy is a refinement of another replacement policy, it is of interest to compare these two block replacement policies. Block et al. (1990) proved that $N^{(b)}(t, \ell) \leq_{\text {st }} N^{(b)}\left(t, \ell^{\prime}\right)$ for $\{k \ell\}_{k=1}^{\infty} \supset\left\{k \ell^{\prime}\right\}_{k=1}^{\infty}$ if and only if $F$ is NBU. In the following theorem, we propose a parallel version for $\mathrm{NBU}_{\mathrm{MG}}$.

Theorem 3.3. Let $N_{i}(t, \ell)$ be the total number of failures accumulated in $[0, t]$ under the policy to replace at $k \ell, k=0,1, \ldots, i$. If the underlying distribution $F$ is $N B U_{M G}$ then $N_{i}(t, \ell) \geq_{\mathrm{MGF}} N_{j}(t, \ell)$ for any integers $j \geq i \geq 1$. 
Proof. It is evident that we only need to prove that $N_{i}(t, \ell) \geq_{\mathrm{MGF}} N_{i+1}(t, \ell)$, or, equivalently, for any $s \geq 0$ and $n=1,2, \ldots$,

$$
\int_{0}^{\infty} \mathrm{e}^{s t} \mathrm{P}\left(N_{i}(t, \ell)<n\right) \mathrm{d} t \leq \int_{0}^{\infty} \mathrm{e}^{s t} \mathrm{P}\left(N_{i+1}(t, \ell)<n\right) \mathrm{d} t .
$$

Note that $N_{i}(t, \ell) \stackrel{\text { st }}{=} N_{i+1}(t, \ell)$ for $t \leq(i+1) \ell$. To obtain (3.3), it suffices to prove that

$$
\int_{(i+1) \ell}^{\infty} \mathrm{e}^{s t} \mathrm{P}\left(N_{i}(t, \ell)<n\right) \mathrm{d} t \leq \int_{(i+1) \ell}^{\infty} \mathrm{e}^{s t} \mathrm{P}\left(N_{i+1}(t, \ell)<n\right) \mathrm{d} t .
$$

Let $T_{i, n}$ be the $n$th failure time corresponding to $N_{i}(t, \ell)$. Given $N_{i}((i+1) \ell, \ell)=j$, denote by $U_{j+1}, j=0,1, \ldots, n-1$, the interval between $(i+1) \ell$ and $T_{i, j+1}$, i.e.

$$
U_{j+1}=\left[T_{i, j+1}-(i+1) \ell \mid N_{i}((i+1) \ell, \ell)=j\right] .
$$

For $t>(i+1) \ell, N_{i+1}(t, \ell)-N_{i+1}((i+1) \ell, \ell)$ is just a renewal process with $(i+1) \ell$ unit time shifted from the origin. We have

$$
\begin{gathered}
\int_{(i+1) \ell}^{\infty} \mathrm{e}^{s t} \mathrm{P}\left(N_{i+1}(t, \ell)<n \mid N_{i+1}((i+1) \ell, \ell)=j\right) \mathrm{d} t \\
=\int_{(i+1) \ell}^{\infty} \mathrm{e}^{s t} \mathrm{P}\left(\tau_{j+1}+\cdots+\tau_{n}>t-(i+1) \ell\right) \mathrm{d} t \\
=\mathrm{e}^{s(i+1) \ell} \int_{0}^{\infty} \mathrm{e}^{s t} \mathrm{P}\left(\tau_{j+1}+\cdots+\tau_{n}>t\right) \mathrm{d} t .
\end{gathered}
$$

Furthermore, by conditioning on $N_{i}((i+1) \ell, \ell)=N_{i+1}((i+1) \ell, \ell)$, it holds that

$$
\begin{aligned}
\int_{(i+1) \ell}^{\infty} & \mathrm{e}^{s t} \mathrm{P}\left(N_{i+1}(t, \ell)<n\right) \mathrm{d} t \\
= & \sum_{j=0}^{n-1} \mathrm{P}\left(N_{i+1}((i+1) \ell, \ell)=j\right) \mathrm{e}^{s(i+1) \ell} \int_{0}^{\infty} \mathrm{e}^{s t} \mathrm{P}\left(\tau_{j+1}+\cdots+\tau_{n}>t\right) \mathrm{d} t .
\end{aligned}
$$

Similarly,

$$
\begin{aligned}
\int_{(i+1) \ell}^{\infty} & \mathrm{e}^{s t} \mathrm{P}\left(N_{i}(t, \ell)<n \mid N_{i}((i+1) \ell, \ell)=j\right) \mathrm{d} t \\
= & \int_{(i+1) \ell}^{\infty} \mathrm{e}^{s t} \mathrm{P}\left((i+1) \ell+U_{j+1}+\tau_{j+2}+\cdots+\tau_{n}>t\right) \mathrm{d} t \\
= & \mathrm{e}^{s(i+1) \ell} \int_{0}^{\infty} \mathrm{e}^{s t} \mathrm{P}\left(U_{j+1}+\tau_{j+2}+\cdots+\tau_{n}>t\right) \mathrm{d} t
\end{aligned}
$$

and

$$
\begin{aligned}
\int_{(i+1) \ell}^{\infty} & \mathrm{e}^{s t} \mathrm{P}\left(N_{i}(t, \ell)<n\right) \mathrm{d} t \\
\quad= & \sum_{j=0}^{n-1} \mathrm{P}\left(N_{i}((i+1) \ell, \ell)=j\right) \mathrm{e}^{s(i+1) \ell} \int_{0}^{\infty} \mathrm{e}^{s t} \mathrm{P}\left(U_{j+1}+\tau_{j+2}+\cdots+\tau_{n}>t\right) \mathrm{d} t .
\end{aligned}
$$


Now, it is evident that (3.4) is equivalent to

$$
\int_{0}^{\infty} \mathrm{e}^{s t} \mathrm{P}\left(U_{j+1}+\tau_{j+2}+\cdots+\tau_{n}>t\right) \mathrm{d} t \leq \int_{0}^{\infty} \mathrm{e}^{s t} \mathrm{P}\left(\tau_{j+1}+\cdots+\tau_{n}>t\right) \mathrm{d} t .
$$

That is,

$$
U_{j+1}+\tau_{j+2}+\cdots+\tau_{n} \leq_{\mathrm{MGF}} \tau_{j+1}+\tau_{j+2}+\cdots+\tau_{n} .
$$

Owing to Theorem 2.2 of Klar and Müller (2003) again, to prove (3.5), it suffices to prove that $U_{j+1} \leq_{\mathrm{MGF}} \tau_{j+1}$, which is equivalent to

$$
\begin{aligned}
& \int_{0}^{(i+1) \ell}\left[\int_{0}^{\infty} \mathrm{e}^{s t} \mathrm{P}\left(U_{j+1} \geq t \mid T_{i, j}=u\right) \mathrm{d} t\right] \mathrm{dP}\left(T_{i, j} \leq u \mid N_{i}((i+1) \ell, \ell)=j\right) \\
& \quad \leq \int_{0}^{(i+1) \ell}\left[\int_{0}^{\infty} \mathrm{e}^{s t} \mathrm{P}\left(X \geq t \mid T_{i, j}=u\right) \mathrm{d} t\right] \mathrm{dP}\left(T_{i, j} \leq u \mid N_{i}((i+1) \ell, \ell)=j\right) .
\end{aligned}
$$

Note that

$$
\left(U_{j+1} \mid T_{i, j}=u\right) \stackrel{\text { st }}{=} \begin{cases}X_{\ell}=(X-\ell \mid X \geq \ell) & \text { if } u \leq i \ell \\ X_{(i+1) \ell-u} & \text { if } i \ell<u \leq(i+1) \ell .\end{cases}
$$

By the $\mathrm{NBU}_{\mathrm{MG}}$ property of $X$, we immediately have

$$
\left(U_{j+1} \mid T_{i, j}=u\right) \leq_{\mathrm{MGF}} X .
$$

This validates (3.6) and, hence, $U_{j+1} \leq_{\text {MGF }} \tau_{j+1}$. Thus, we reach the desired conclusion.

In the literature, some authors have investigated the comparison between the age replacement policy and the corresponding renewal process. For example, Marshall and Proschan (1972) showed that $F$ is NBU if and only if $N(t) \geq_{\text {st }} N^{(a)}(t, \ell)$ for any $t \geq 0$ and $\ell \geq 0$. Yue and Cao (2001) proved that $F$ is $\mathrm{NBU}_{\mathrm{LT}}$ if and only if $\tau_{i} \leq_{\mathrm{Lt}} \tau_{i}^{(a)}$ for $i=0,1,2, \ldots$. Belzunce $e t$ al (2005) deduced that $N(t) \leq_{\text {icx }} N^{(a)}(t, \ell)$ for any $t \geq 0$ and $\ell \geq 0$ if $F$ is NBUC. Recently, Belzunce $e t$ al. (2006) proved that $F$ is NBUE if and only if $\mathrm{E} T_{i} \leq \mathrm{E} T_{i}^{(a)}$ for $i=1,2, \ldots$. A parallel version for $\mathrm{NBU}_{\mathrm{MG}}$ life distributions is presented as Theorem 3.4 below, which states that the age replacement policy reduces the number of failures of an $\mathrm{NBU}_{\mathrm{MG}}$ unit in the sense of the MGF order.

Theorem 3.4. The underlying life distribution $F$ is $N B U_{M G}$ if and only if $N(t) \geq_{\mathrm{MGF}} N^{(a)}(t, \ell)$ for all $t, \ell \geq 0$.

Proof. Necessity. Suppose that $F$ is $\mathrm{NBU}_{\mathrm{MG}}$. By Definition 3.1 we need to prove that

$$
\sum_{i=1}^{n} \tau_{i}=T_{n} \leq_{\mathrm{MGF}} T_{n}^{(a)}=\sum_{i=1}^{n} \tau_{i}^{(a)} \quad \text { for } n=1,2, \ldots
$$

Owing to Theorem 2.2 of Klar and Müller (2003), this boils down to $\tau_{i} \leq_{\mathrm{MGF}} \tau_{i}^{(a)}$ for all $i=1,2, \ldots$. In view of the fact that

$$
\mathrm{P}\left(\tau_{i}^{(a)}>t\right)=\bar{F}^{k}(\ell) \bar{F}(t-k \ell) \quad \text { for } k \ell \leq t<(k+1) \ell \text { and } k=0,1,2, \ldots,
$$


we can prove that $\tau_{i} \leq_{\mathrm{MGF}} \tau_{i}^{(a)}$ for all $i=1,2, \ldots$ following the same lines as in the proof of Theorem 3.2.

Sufficiency. Suppose that $N(t) \geq_{\mathrm{MGF}} N^{(a)}(t, \ell)$ for any $t \geq 0$. Then, for any $s \geq 0$,

$$
\int_{0}^{\infty} \mathrm{e}^{s t} \mathrm{P}(N(t)=0) \mathrm{d} t \leq \int_{0}^{\infty} \mathrm{e}^{s t} \mathrm{P}\left(N^{(a)}(t, \ell)=0\right) \mathrm{d} t .
$$

Note that $\mathrm{P}(N(t)=0)=\bar{F}(t)$ for any $t \geq 0$ and

$$
\mathrm{P}\left(N^{(a)}(t, \ell)=0\right)=\bar{F}^{k}(\ell) \bar{F}(t-k \ell) \quad \text { for } k \ell \leq t<(k+1) \ell \text { and } k=0,1,2, \ldots
$$

Following the same lines as in the proof of Theorem 3.2, we can prove that $F$ is $\mathrm{NBU}_{\mathrm{MG}}$.

Combining Theorem 3.1 with Theorem 3.4, we immediately establish the following relation between the age replacement policy and the minimal repair policy.

Corollary 3.2. If the underlying distribution $F$ is $N B U_{M G}$ then $N^{(m)}(t) \geq_{\mathrm{MGF}} N^{(a)}(t, \ell)$ for all $t, \ell \geq 0$.

\section{Acknowledgements}

The authors would like to thank the constructive comments of two anonymous referees.

\section{References}

Barlow, R. E. and Proschan, F. (1981). Statistical Theory of Reliability and Life Testing. Holt, New York.

Belzunce, F., Ortega, E. And Ruiz, J. M. (1999). The Laplace order and ordering of residual lives. Statist. Prob. Lett. 42, 145-156.

Belzunce, F., Ortega, E.-M. And Ruiz, J. M. (2005). A note on replacement policy comparisons from NBUC lifetime of the unit. Statist. Papers 46, 509-522.

Belzunce, F., Ortega, E.-M. And Ruiz, J. M. (2006). Comparison of expected failure times for several replacement policies. IEEE Trans. Reliab. 55, 490-495.

Block, H. W., Langberg, N. A. And Savits, T. H. (1990). Maintenance comparisons: block policies. J. Appl. Prob. 27, 649-657.

Block, H. W., Langberg, N. A. and Savits, T. H. (1993). Repair replacement policies. J. Appl. Prob. 30, $194-206$.

KLAR, B. (2002). A note on the $\mathcal{L}$-class of life distributions. J. Appl. Prob. 39, 11-19.

KLAR, B. AND MüLlER, A. (2003). Characterizations of classes of lifetime distributions generalizing the NBUE class. J. Appl. Prob. 40, 20-32.

KLEFsJö, B. (1983). A useful ageing property based on the Laplace transform. J. Appl. Prob. 20, 615-626.

LAI, C.-D. AND XIE, M. (2006). Stochastic Ageing and Dependence for Reliability. Springer, New York.

LI, X. (2004). Some properties of aging notions based on the moment-generating-function order. J. Appl. Prob. 41, 927-934.

Marshall, A. W. and Olkin, I. (2007). Life Distributions. Springer, New York.

Marshall, A. W. and Proschan, F. (1972). Classes of distributions applied in replacement, with renewal theory implications. In Proc. 6th Berkeley Symp. Math. Statistics Prob., Vol. I, University of California Press, Berkeley, CA, pp. 395-415.

Müller, A. And Stoyan, D. (2002). Comparison Methods for Stochastic Models and Risks. John Wiley, Chichester. Shaked, M. and Shanthikumar, J. G. (2007). Stochastic Orders. Springer, New York.

WANG, W. Y. (1996). Life distribution classes and two-unit standby redundant system. Doctoral Thesis, Chinese Academy of Sciences.

Yue, D. AND CaO, J. (2001). The NBUL class of life distribution and replacement policy comparisons. Naval Res. Logistics 48, 578-591. 